\title{
LIE THEORETIC PERSPECTIVE OF BLACK-SCHOLES EQUATION UNDER STOCHASTIC HESTON MODEL
}

\author{
Maba Boniface Matadi ${ }^{1}$, Phumlani Lawrence Zondi $^{2}$ \\ ${ }^{1}$ University of Zululand, Department of Mathematical Science \\ Private Bag X1001 \\ KwaDlangeZwa - 3886, SOUTH AFRICA \\ ${ }^{2}$ University of Zululand, Department of Mathematical Science \\ Private Bag X1001 \\ KwaDlangeZwa - 3886, SOUTH AFRICA
}

\begin{abstract}
This study examines a classical Black-Scholes (BC) model for stochastic volatility with Heston process from Lie symmetry perspective. In the same way the study includes a classification of point symmetries and the corresponding modified local one-parameter transformations. Lie symmetry analysis is presented for the case where the volatility is a stochastic process. Furthermore, an invariant solutions are calculated and illustrated numerically.
\end{abstract}

AMS Subject Classification: 34C14, 34M55

Key Words: group theoretic approach; Lie symmetry; invariant solution; Black-Scholes equation

\section{Introduction}

In this paper, the Lie symmetry analysis of the BS model with stochastic volatility presented in the paper by Paliathanasis et al in [12] is brought into perspec-

Received: March 5, 2020

(C) 2020 Academic Publications

$\S_{\text {Correspondence author }}$ 
tive. In particular, We venture to give a self-contained (detailed ) exposition of their paper. We point out that there was a slight error in their calculations that led to a wrong reduced differential equation. We then provide a new correct differential equation and then draw its numerical solutions.

The generalized Heston model is a slight variation of the Orstein-Uhlenbeck model. The Heston model is obtained by letting $f(y)=\sqrt{y}$ and $\beta=\rho \sqrt{y}$ in the Orstein-Uhlenbeck model, to acquire the given stochastic differential equation $[2]$

$$
\begin{aligned}
& d S=r S d t+\sqrt{y} S d W^{s}, \\
& d \sigma=\alpha(m-y) d t+\delta \sqrt{y} d Z^{\sigma},
\end{aligned}
$$

where $W^{s}$ is the Brownian motion of the stock price; $Z^{\sigma}$ is Brownian motion of the volatility of the stock; $\alpha$ is the rate of mean-reversion; $m$ is the long-run mean of $Y ; r$ is the drift of $Z$ and $\delta$ the correlation coefficient. The term $\sqrt{y}$ guarantees the positive volatility. Heston model is the most famous of all the stochastic volatility models; hence, much of the analysis and benchmarks are done using it. The differential equation resulting from this model is given by $[9]$

$$
\begin{array}{r}
V_{t}+\frac{1}{2} S^{2} y V_{S S}+\rho \delta y S V_{S y}+\frac{1}{2} \delta^{2} y V_{y y}+r S V_{S} \\
+(\alpha m-(\alpha+\lambda) y) V_{y}-r V=0 .
\end{array}
$$

Lie groups are mathematical objects that depicts properties of groups as they are known in group theory. The idea behind Lie group theory is to apply suitable transformations of independent and dependent variables to obtain a Lie symmetries. This process results into differential equations with reduced orders compared to the original equation [11]. Transformations of this kind are known as infinitesimal transformations. The important feature of Lie groups is the concept of infinitesimal generators. Infinitesimal generators are obtained by solving a symmetry condition of the symmetry group [3]. Ultimately, working from the infinitesimal generators symmetries of differential equations can be generated. Lie provided a very comprehensive classification of differential equations. According to this classification, all parabolic equations admitting the symmetry group of highest order reduce to the heat conduction equation [10]. This is where the theory of Lie group analysis connects with the BS model, as it is also transformable into the heat equation. Lie's theory have thus proved useful in facilitating analysis for option pricing using BS model.

In this paper, the BS equation following a stochastic volatility of Heston type is considered. This equation is subjected to an infinitesimal generator 
whose coefficients are put into a determining equation. However, the determining equation is very complicated to obtained. Therefore, a Symbolic (SYM) Package (see [1]) of Mathematica is used and it resulted into four symmetries (including linear and infinite symmetries). Linear combinations of these symmetries are used as symmetry vectors. The characteristic systems of these vectors are constructed and integrated and led to invariants which reduced the original equation into a second-order linear ordinary differential equation. The solution of this equation is calculated using Maple package and numerical solutions are depicted for different parameter values.

This paper is organised as follows. In Section 2, a background of the concepts underlying the theory of Lie symmetry analysis are introduced. The Symmetry Analysis of Heston Model is carry out and obtained an invariant solutions in Section 3. The numerical solutions is performed and presented graphically in Section 4.

\section{Lie symmetry analysis}

\subsection{Fundamental definitions and theorems}

In this section, a comprehensive review of a group theoretic approach to the solution of differential equations is given. The theory entails the tools necessary for subsequent to be employed throughout the paper. To start with, the mathematical idea of a symmetry is explained, and then the general properties of groups are explained; the properties are then extended to the Lie groups.

The $k$ th-order differential equation [5]

$$
u_{t}-F\left(t, x, u, u_{(1)}, u_{(2)}, \ldots, u_{(k)}\right)=0,
$$

admits the given Lie group of transformations of one-parameter

$$
\begin{aligned}
\hat{t} & \approx t+a \xi^{0}\left(t, x, u, u_{(1)}, u_{(2)}, \ldots, u_{(k)}\right) \\
\hat{x}^{i} & \approx x^{i}+a \xi^{i}\left(t, x, u, u_{(1)}, u_{(2)}, \ldots, u_{(k)}\right) \\
\hat{u}_{i}^{\alpha} & \approx u_{i}^{\alpha}+a \eta_{i}^{\alpha}\left(t, x, u, u_{(1)}, u_{(2)}, \ldots, u_{(k)}\right)
\end{aligned}
$$

with infinitesimal Lie generator [6]

$$
X=\xi^{0} \frac{\partial}{\partial t}+\xi^{i} \frac{\partial}{\partial x^{i}}+\eta^{\alpha} \frac{\partial}{\partial u^{\alpha}},
$$

if 


$$
\hat{u}_{t}-F\left(\hat{t}, \hat{x}, \hat{u}, \hat{u}_{(1)}, \hat{u}_{(2)}, \ldots, \hat{u}_{(k)}\right)=0 .
$$

The group transformations $\hat{t}, \hat{x}$ and $\hat{u}$ are obtained by solving the following Lie equations [8]

$$
\begin{aligned}
\frac{d \hat{t}}{d a} & =\xi^{0}\left(\hat{t}, \hat{x}, \hat{u}, \hat{u}_{(1)}, \hat{u}_{(2)}, \ldots, \hat{u}_{(k)}\right) \\
\frac{d \hat{x}^{i}}{d a} & =\xi^{i}\left(\hat{t}, \hat{x}, \hat{u}, \hat{u}_{(1)}, \hat{u}_{(2)}, \ldots, \hat{u}_{(k)}\right) \\
\frac{d \hat{u}_{i}^{\alpha}}{d a} & =\eta_{i}^{\alpha}\left(\hat{t}, \hat{x}, \hat{u}, \hat{u}_{(1)}, \hat{u}_{(2)}, \ldots, \hat{u}_{(k)}\right)
\end{aligned}
$$

with initial conditions

$$
\left.\hat{t}\right|_{a=0}=t,\left.\hat{x}^{i}\right|_{a=0}=x^{i},\left.\hat{u}_{i}^{\alpha}\right|_{a=0}=u_{i}^{\alpha} .
$$

The infinitesimal form of $\hat{u}_{\bar{t}}, \hat{u}_{(1)}, \hat{u}_{(2)}, \ldots, \hat{u}_{(k)}$ are found by the given formulas [7]:

$$
\begin{aligned}
\hat{u}_{i}^{\alpha} & \approx u_{i}^{\alpha}+a \eta_{i}^{\alpha}\left(x, u, u_{1}\right) \\
\hat{u}_{i j}^{\alpha} & \approx u_{i j}^{\alpha}+a \eta_{i j}^{\alpha}\left(x, u, u_{1}, u_{2}\right) \\
& \ldots \\
\hat{u}_{i_{1} \ldots i_{k}}^{\alpha} & \approx u_{i_{1} \ldots i_{k}}^{\alpha}+a \eta_{i_{1} \ldots i_{k}}^{\alpha}\left(x, u, u_{1}, \ldots, u_{k}\right) .
\end{aligned}
$$

The functions $\eta_{i}^{\alpha}\left(x, u, u_{1}\right), \eta_{i j}^{\alpha}\left(x, u, u_{1}, u_{2}\right)$, and $\eta_{i_{1} \ldots i_{k}}^{\alpha}\left(x, u, u_{1}, \ldots, u_{k}\right)$ are obtained from the following prolongation formulas [3]

$$
\begin{aligned}
\eta_{i}^{\alpha} & =D_{i}\left(\eta^{\alpha}\right)-u_{j}^{\alpha} D_{i}\left(\xi^{j}\right) \\
\eta_{i j}^{\alpha} & =D_{j}\left(\eta_{i}^{\alpha}\right)-u_{i l}^{\alpha} D_{j}\left(\xi^{l}\right) \\
& \cdots \\
\eta_{i_{1} \ldots i_{k}}^{\alpha} & =D_{i_{k}}\left(\eta_{i_{1} \ldots i_{k-1}}^{\alpha}\right)-u_{i_{1} \ldots i_{k-1} l}^{\alpha} D_{i_{k}}\left(\xi^{l}\right),
\end{aligned}
$$

where $D_{i}$ denotes the operator of total differentiation with respect to $\left(x_{1}, x_{2} \ldots x_{n}\right)$, then

$$
D_{i}=\frac{\partial}{\partial x_{i}}+u_{i}^{\alpha} \frac{\partial}{\partial u^{\alpha}}+u_{i j}^{\alpha} \frac{\partial}{\partial u_{j}^{\alpha}} .
$$

The transformed derivatives $\hat{u}_{(1)}, \hat{u}_{(2)}, \ldots, \hat{u}_{(k)}$ can be computed from the formulae 


$$
D_{i}=D_{i}\left(f^{i}\right) \hat{D}_{j}
$$

The generators are therefore given by

$$
\begin{aligned}
X^{[1]} & =X+\eta_{i}^{\alpha}\left(x, u, u_{1}\right) \frac{\partial}{\partial u_{i}^{\alpha}} \\
& \ldots \\
X^{[k]} & =X^{[1]}+\ldots+\eta_{i_{1} \ldots i_{k}}^{\alpha}\left(x, u, \ldots, u_{k}\right) \frac{\partial}{\partial u_{i_{1} \ldots i_{k}}^{\alpha}} .
\end{aligned}
$$

Theorem 1. A function $F\left(x, u, \cdots, u_{k}\right)$ is invariant under the prolonged group $G$, if and only if [4]

$$
X^{[k]} F=0
$$

where $X^{[k]}$ is the generator of $G$.

Theorem 2. Every one-parameter group of transformations $(\hat{x}=f(x, y, \varepsilon)$, $\hat{y}=g(x, y, \varepsilon))$ is reduced to a group of translations $\hat{t}=t+\varepsilon, \hat{u}=u$ with the generator [4]

$$
X=\frac{\partial}{\partial t}
$$

by suitable change of variables

$$
t=t(x, y), \quad u=u(x, y)
$$

The overall idea behind the Lie theory is to transform independent and dependant variable of a differential equation, such that the order of a deferential equation gets reduced and easy to solve. If a differential equation admits the symmetry group, its order can be reduced by using what is called Canonical Coordinates.

Definition 3. ([3]) The canonical coordinates $(r(x, y), s(x, y))$ of a differential equation are the coordinates in which the equation becomes separable. 


\subsection{The rational behind Lie point symmetries of partial differential equations}

The BS equation (3) is a partial differential equation that consists of one dependent variable $V$ and two independent variables $t$ and $S$. This section shows how to deal with PDEs of this kind. The result obtained here will be used in subsequent sections to find Lie symmetries for equation (3). In the case of two dependent variables, the point transformation are of the form

$$
\Gamma:(x, t, u) \mapsto(\hat{x}(x, t, u)), \hat{t}(x, t, u), \hat{u}(x, t, u) .
$$

Therefore, the goal is to seek point symmetries of the form $[5,7]$

$$
\begin{gathered}
\hat{x}=x+\varepsilon \xi(x, t, u)+O\left(\varepsilon^{2}\right), \\
\hat{t}=t+\varepsilon \tau(x, t, u)+O\left(\varepsilon^{2}\right), \\
\hat{u}=u+\varepsilon \eta(x, t, u)+O\left(\varepsilon^{2}\right) .
\end{gathered}
$$

The corresponding infinitesimal generator is given by $[6,8]$

$$
X=\xi \partial_{x}+\tau \partial_{t}+\eta \partial_{u}
$$

The first and the second prolongations of (16) are

$$
\begin{aligned}
& X^{[1]}=\xi \partial_{x}+\tau \partial_{t}+\eta \partial_{u}+\eta_{x}^{(1)} \partial_{u_{x}}+\eta_{t}^{(1)} \partial_{u_{t}}, \\
& X^{[2]}=X^{[1]}+\eta_{x x}^{2} \partial_{x x}+\eta_{x t}^{(2)} \partial_{u_{x t}}+\eta_{t t}^{(2)} \partial_{u_{t t}} .
\end{aligned}
$$

Define the following PDE as

$$
\triangle\left(x, t, u, u_{x}, u_{t}, u_{x x}, u_{t t},\right)=0 .
$$

The symmetry condition is given by

$$
\triangle\left[\hat{x}, \hat{t}, \hat{u}, \hat{u_{x}}, \hat{u_{t}}, \hat{u_{x x}}, \hat{u_{t t}}\right]=0
$$

when (19) holds. This symmetry condition can be differentiated with respect to the parameter $\epsilon$ at $\epsilon=0$ to obtain the linearised symmetry condition $[3,5]$

$$
X^{[2]} \triangle\left[\hat{x}, \hat{t}, \hat{u}, \hat{u_{x}}, \hat{u_{t}}, \hat{u_{x x}}, \hat{u_{t t}}\right]=0,
$$

when

$$
\triangle\left[x, t, u, u_{x}, u_{t}, u_{x x}, u_{t t}\right]=0 .
$$




\section{Symmetry analysis for the Heston model}

This section is intended at performing Lie symmetry analysis for the Heston model. As it was seen in the introduction, the Black-Scholes equation for the Heston model is given by

$$
\begin{aligned}
0= & \frac{1}{2} Y S^{2} V_{S S}+\rho \delta Y S V_{S Y}+\frac{1}{2} \delta^{2} Y V_{Y Y}+r S V_{S} \\
& +(\alpha(m-Y)-\lambda Y) V_{Y}-r V+V_{t} .
\end{aligned}
$$

In order to ease the process of calculating symmetries for equation (23), the following change of variables is employed

$$
\begin{aligned}
Y & =y^{2}, \\
\beta & =\frac{\delta}{2}, \\
c_{1} & =(\alpha-\lambda), \\
c_{2} & =\alpha m-\beta^{2},
\end{aligned}
$$

which gives the new differential equation below:

$$
\begin{aligned}
0 & =\frac{1}{2} y^{2} S^{2} V_{S S}+\beta \rho y S V_{S y}+\frac{1}{2} \beta^{2} V_{y y}+r S V_{S} \\
& +\frac{1}{2}\left[c_{1} y+\left(\frac{c_{2}}{y}\right)\right] V_{y}-r V+V_{t} .
\end{aligned}
$$

Using the SYM, this equation has the determining system of equations given by

$$
\begin{aligned}
& X_{1}=\partial_{t}, \\
& X_{2}=S \partial_{S}, \\
& X_{V}=V \partial_{V}, \\
& X_{b}=b(t, S, y) \partial_{V},
\end{aligned}
$$

where $X_{V}$ and $X_{b}$ are linear symmetry and infinite symmetry, respectively. Now, consider the linear combinations of these symmetries to obtain

$$
\begin{aligned}
& Y_{1}=X_{1}+k_{1} X_{v}, \\
& Y_{2}=X_{2}+k_{2} X_{V}, \\
& Y_{12}=X_{1}+c X_{2}+k_{3} X_{V} .
\end{aligned}
$$




\subsection{Invariant solution through symmetry $Y_{1}$}

The symmetry $Y_{1}$ is given by

$$
Y_{1}=\partial_{t}+k_{1} V \partial_{V}
$$

The characteristic system is therefore given by

$$
\frac{k_{1} d t}{1}=\frac{d V}{V} .
$$

Integrating both sides gives

$$
k_{1} t=\ln V+\ln \Phi(S, y),
$$

which results into

$$
V(t, S, y)=\Phi(S, y) e^{k_{1} t} .
$$

Now, computing the partial derivatives of $V$ with respect to its parameters gives

$$
\begin{aligned}
& V_{t}=k_{1} \Phi(S, y) e^{k_{1} t}, \\
& V_{y}=\Phi_{y} e^{k_{1} t} \\
& V_{S}=\Phi_{S} e^{k_{1} t} \\
& V_{S S}=\Phi_{S S} e^{k_{1} t} \\
& V_{S y}=\Phi_{S y} e^{k_{1} t} \\
& V_{y y}=\Phi_{y y} e^{k_{1} t}
\end{aligned}
$$

Substituting these in equation (25), the function $\Phi(S, y)$ satisfies the following equation

$$
\begin{aligned}
0 & =\frac{1}{2} y^{2} S^{2} \Phi_{S S}+\beta \rho y S \Phi_{S y}+\frac{1}{2} \beta^{2} \Phi_{y y} \\
& +\frac{1}{2}\left[c_{1} y+\left(\frac{c_{2}}{y}\right)\right] \Phi_{y}-r \Phi+k_{1} \Phi .
\end{aligned}
$$

Using Mathematica SYM package [1], it is found that equation (33) admits one symmetry $X_{2}=S \partial_{S}$. This means that if the symmetry $S \partial_{S}+k_{2} V d V$, which is equivalent to the symmetry vector $Y_{2}$ is applied to equation (33) it will be able to reduce the equation into a second-order ordinary differential equation. This is shown below in details. 


\subsection{Invariant solution through symmetry $X_{2}$}

The symmetry $Y_{2}$ is given by

$$
Y_{2}=S \partial_{S}+k_{2} \Phi \partial_{\Phi}
$$

which gives the following characteristic system

$$
k_{2} \frac{d S}{S}=\frac{d \Phi}{\Phi} .
$$

Integrating both sides gives

$$
k_{2} \ln S+\ln W(y)=\ln \Phi,
$$

i.e.

$$
\ln S^{k_{2}}+\ln W(y)=\ln \Phi,
$$

and therefore

$$
\Phi(S, y)=S^{k_{2}} W(y) .
$$

Substituting equation (38) where there is $\Phi$ in equation (31) gives

$$
V(t, S, y)=e^{k_{1} t} S^{k_{2}} W(y) .
$$

One can use the equation (38) to reduce equation (33) by computing partial derivatives of $\Phi$ with respect to $S$ and $y$ as follows

$$
\begin{aligned}
& \Phi_{y}=S^{k_{2}} W_{y}, \\
& \Phi_{S}=k_{2} S^{k_{2}-1} W, \\
& \Phi_{S y}=k_{2} S^{k_{2}-1} W_{y}, \\
& \Phi_{S S}=k_{2}\left(k_{2}-1\right) S^{k_{2}-2} W, \\
& \Phi_{y y}=S^{k_{2}} W_{y y} .
\end{aligned}
$$

Substituting these partial derivatives into equation (33) gives

$$
\begin{aligned}
0= & \beta^{2} W_{y y}+\left(2 \beta \rho y k_{2}+c_{1} y+\frac{c_{2}}{y}\right) W_{y}+\left(2 k_{1}-2 r\left(1-k_{2}\right)\right. \\
& \left.+y^{2}\left(k_{2}^{2}-k_{2}\right)\right)
\end{aligned}
$$

which is the correct version of the linear differential equation obtained in [12]. 


\section{Numerical Solutions}

The new numerical solutions for this differential equation were computed using Maple software and they are depicted in graphs below.

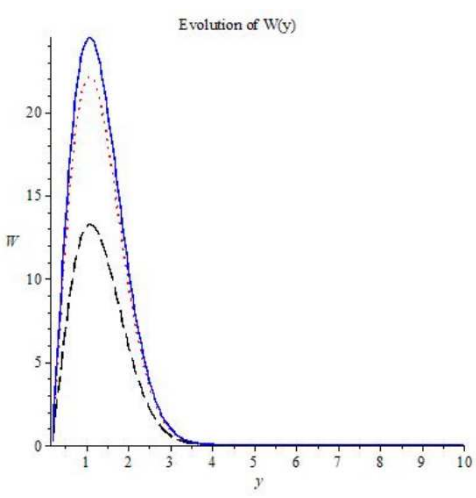

Figure 1: Numerical Solution of the invariant solution for the Heston model equation (41), parameters are chosen as follows:

- Black dashed line: $\rho=0.5, \beta=0.7, k_{1}=1, k_{2}=0.5, r=0.5, c_{1}=-0.01$, and $c_{2}=-0.05$.

- Solid blue line: $\rho=0.5, \beta=0.7, k_{1}=1, k_{2}=0.5, r=0.5, c_{1}=-0.01$, and $c_{2}=-0.003$.

- Red dotted line: $\rho=0.5, \beta=0.7, k_{1}=1, k_{2}=0.5, r=0.5, c_{1}=-0.01$, and $c_{2}=-0.01$

\section{Conclusion}

This study began by looking at the evolution of the solution of the Black-Scholes model for stochastic volatility using the technique known as the modified local one-parameter transformations. Symmetries were obtained and two of them were used to obtain an invariant solution. The future work in this regard will involve finding invariant solutions for all the symmetries, and then using these solutions to obtain optimal system of sub-algebras of the model.

The study further looked at the evolution of the solution of the Black- 


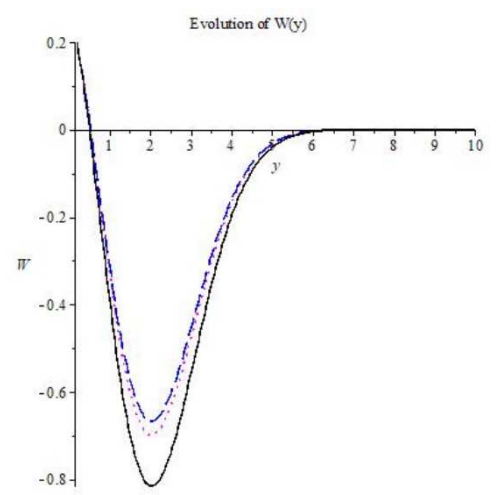

Figure 2: Numerical Solution of the invariant solution for the Heston model equation (41), parameters are chosen as follows:

- Black solid line: $\rho=0.5, \beta=-0.7, k_{1}=1, k_{2}=0.5, r=0.5, c_{1}=-0.01$, and $c_{2}=-0.05$.

- Blue dashed line: $\rho=0.5, \beta=-0.7, k_{1}=1, k_{2}=0.5, r=0.5, c_{1}=$ -0.01 , and $c_{2}=0.003$.

- Red dotted line: $\rho=0.5, \beta=-0.7, k_{1}=1, k_{2}=0.5, r=0.5, c_{1}=-0.01$, and $c_{2}=-0.01$.

Scholes model for stochastic volatility, the model was assumed to follow the Heston process, and the Lie symmetry analysis reduced the model to a secondorder ordinary differential equation. The future work in this regard will be to incorporate the dividend yield and observe how the solutions evolve. Another possible extension of the model is to consider an interest rate that is not constant, as an instance interest rate can be considered to be a function of time or a stochastic process.

\section{Acknowledgment}

MB Matadi thanks the research office of the University of Zululand for the financial support. 


\section{References}

[1] S. Dimas, D. Tsoubelis, SYM:A new symmetry-finding package for Mathematica, Group Analysis of Differential Equations (2005), 64-70.

[2] S.L. Heston, A closed-form solution for options with stochastic volatility with applications to bonds and currency options, The Review of Financial Studies, 6 (1993), 327-343.

[3] P.E. Hydon, Symmetry Methods for Differential Equations - A Beginner's Guide, Cambridge Texts in Applied Mathematics (2000), E Book (2010).

[4] T.P. Masebe, A Lie symmetry analysis of the Black-scholes Merton finance model through modified local one-parameter transformations, Doctoral Thesis, University of South Africa (2014).

[5] M.B. Matadi, Singularity and Lie group analyses for tuberculosis with exogenous reinfection, International Journal of Biomathematics, 8 (2015), $1-12$.

[6] M.B. Matadi, Lie Symmetry Analysis Of Early Carcinogenesis Model, Applied Mathematics E-Notes 18 (2018), 238-249.

[7] M.B. Matadi, The conservative form of tuberculosis model with demography, Far East Journal of Mathematical Sciences, 102 (2017), 2403-2416.

[8] M.B. Matadi, Symmetry and conservation laws for tuberculosis model, International Journal of Biomathematics, 10 (2017), \# 1750042.

[9] J. Merger, A. Borzi, A Lie algebraic and numerical investigation of the Black-Scholes equation with Heston volatility model, J. Generalized Lie Theory Appl., 2 (2016), 2-7.

[10] F. Oliveri, Lie symmetries of Differential equations: Classical results and recent contributions, Symmetry, 2 (2010), 658-706.

[11] P.J. Olver, Application of Lie Groups to Differential Equations, SpringerVerlag, New York (1986).

[12] A. Paliathanasis, K. Krishnakumar, K.M. Tamizhmani, P.G.L. Leach, Lie Symmetry analysis of the Black-Scholes-Merton Mmdel for European options with stochastic volatility, arXiv preprint: arXiv (2015), 1508.06797. 